\title{
A case of a negative DWI MRI within 12 hours of acute ischemic stroke symptoms.
}

\author{
Hyuk Chang, Hakseung Lee, Yunsu Hwang, Youngseo Kim, Hyunyoung Park \\ Department of Neurology, School of Medicine, Wonkwang University Hospital, Iksan, Korea
}

\section{Background \& Significance}

Diffusion weighted image(DWI) in MRI is the most sensitive tool for detection of ischemic stroke, even in 1 hours from first onset. But its sensitivity can not approach to $100 \%$, so the term 'Diffusion-negative stroke' was made. Diffusion- negative stroke has been known to be more frequent in posterior circulation ischemia, mainly in brain stem than in anterior circulation. We present a case of diffusion-negative stroke in anterior circulation, which was confirmed as ischemic stroke in F/U DWI-MRI.

\section{Case}

A 62 years old women arrived to the emergency department with dysarthria with hemiparesis of right upper and lower extremities. Her neurologic Symptoms emerged about 1 hour and 20minutes before the arrival. And the Symptoms were spontaneously resolved just before arrival. Brain CT was normal findings and MRI was done in series. DWI MRI didn't show any significant abnormality. (A) T1, T2, SWI and enhanced T1 images, either. MRA was also normal findings. But only perfusion MRI (TTP) showed subtle minimal perfusion deficit in left basal ganglia(globus pallidus) and corona radiata. (A) She was hospitalized for closed observation for neurogic symptom and treated with oral clopidogrel in accordance with TIA. 3hours after admission, she complained of right sided hemiparesis and slurred speech again. MRC grades were 3 in right upper and lower limbs. Her symptoms continued to the next day morning, so we tried follow up DWI-MRI, which was done at 12 hours after the first onset of acute stroke symptoms. But DWI was negative.(B) And soon her symptoms was resolved again. In the next day(HD\#3), she showed dysarthria and mild right sided hemiparesis(MRC grade 4). We repeated diffusion \& perfusion MRI once more. Finally, DWI showed very subtle high signal intensity in left basal ganglia and corona radiata, which correlated with the perfusion deficit area in previous perfusion MRI. Follow up perfusion MRI showed no significant difference from the previous study, which had showed perfusion deficit in left basal ganglia(globus pallidus) and corona radiata. (C) She was treated with antiplatelet agent under the confirmative diagnosis of lacunar infarction.

\section{Conclusion or Comments}

Diffusion MRI imaging has been noted with the most sensitve and reliable tool for diagonosis of acute ishemic stroke. But this patient did not showed any abnormal finding in DWI. We propose that the DWI can be the most sensitve tool but, even in negative result in DWI, we can not rule out ischemic stroke perfectly. So close monitoring for the patient's neurologic symptoms are necessary and we should be always concerned about DWI negative stroke.

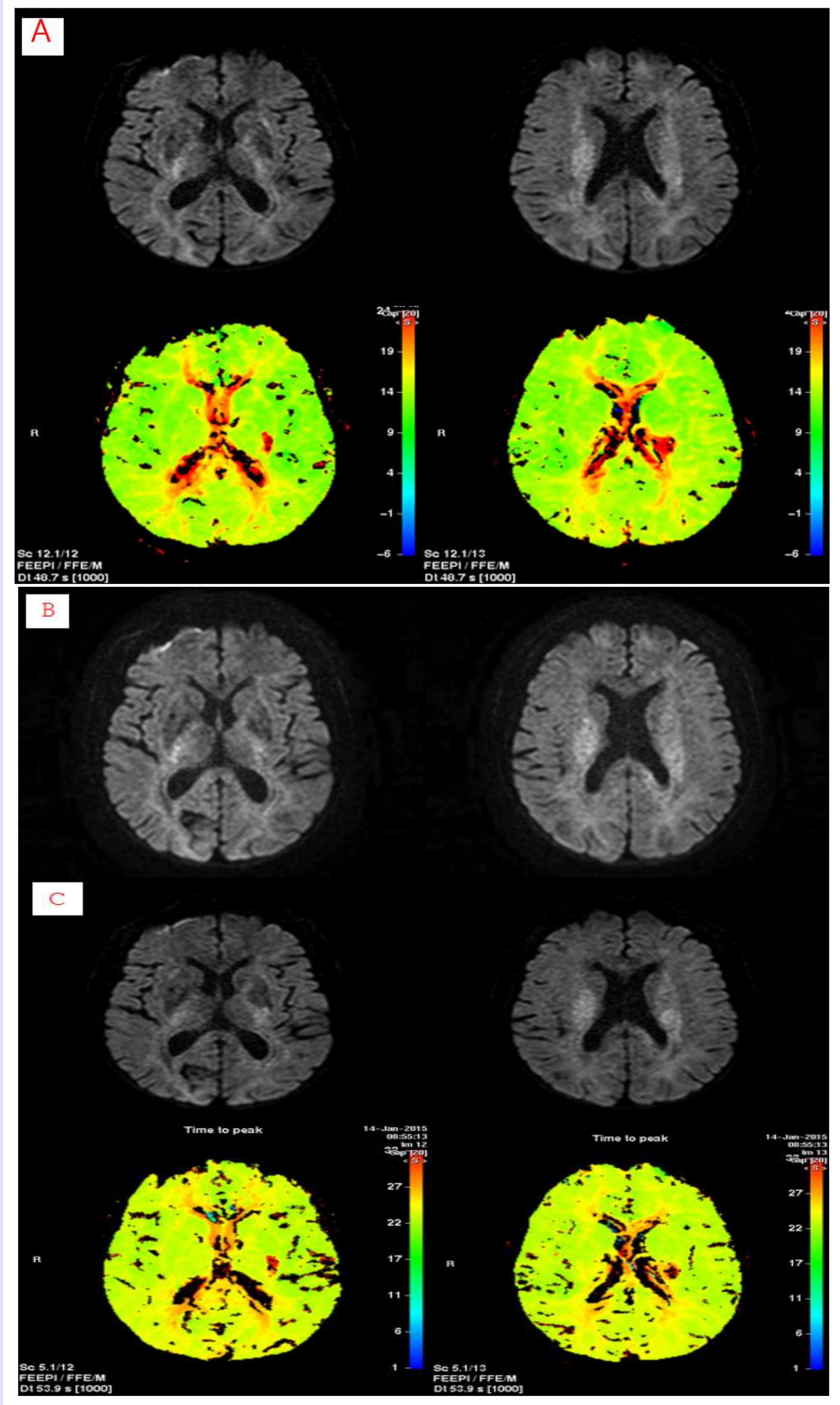

Figure. MRI of the patient. (A) Ininital brain DWI-MRI shows no significant acute brain lesion, but minimal perfusion deficits are seen in left basal ganglia and corona radiate in PWI MRI. (B) Follow up DWI MRI after 12 hours of onset didn't show significant change in contrast to previous study. (C) DWI \& PWI MRI at the $3^{\text {rd }}$ hospital day reveals subtle high signal intensity and perfusion deficit in left basal ganglia and corona radiata, which correlate with the perfusion deficit area in the previous PWI MRI. 